\title{
Fiber Breakage in Spinning Operations
}

\author{
Part 1: Statistical Analysis of the Breaking Process \\ By Hiraku Takeda, Member, TMSJ \\ Faculty of Engineering, Yamagata University, Yomezawa, Yamagata-Ken \\ Based on Journal of the Textile Machinery Society of Japan, Transactions, Vol. 21, No.4, T71-80 (1968)
}

\begin{abstract}
Variations in the fibre length distribution caused by the breakage process, which is assumed to be steady and continuous in time, are discussed generally.

Statistically, such a process is defined by three fractions: (a) the fraction $\alpha(l) \Delta t$ that a fibre of length $l$ breaks in time $\Delta t$; (b) $\beta\left(l_{1} \rightarrow l \Delta l\right.$ that breakage occurs in $l \sim 1 \pm \Delta l$ along a fibre axis having $l_{1}$; and (c) $\gamma(l) \Delta l$ that fibre length $l$ is lost in time $\Delta t$.

From those fractions an integro-differential equation has been derived giving the numberfrequency distribution of fibre lengths at any instant. The equation is soluble by artbitrary functions

Assuming $\gamma=0$, methods to obtain $\alpha$ and $\beta$ experimentally and the upper bound of total number at any instant have been investigated.

By applying the above results to breakage in roller carding, the following estimate of $\alpha$ and $\beta$ has been obtained:

$$
\begin{aligned}
& \alpha(l)=k l^{2.5} \\
& \beta\left(l_{1} \rightarrow l\right)=\frac{6 l}{l_{1}^{2}}\left(1-\frac{l}{l_{1}}\right)
\end{aligned}
$$

Experimental and calculated distributions agree well if this estimate is used.
\end{abstract}

KEY WORDS: FIBER LENGTH, FIBER BREAKAGE, FIBER LENGTH DISTRIBUTION, FREQUENCY DISTRIBUTION (FIBER LENGTH), BREAKING.

\section{Introduction}

The fiber length distribution, one of the major characteristics of spinning material, is not invariable but, instead, varies continuously during spinning operations. Variations in the fiber length distribution are produced mainly two factors:

(1) The elemination of short fibers through the spinning operation, e. g., waste, flyout, etc.

(2) The increase in the number of short fibers due to fiber breakage during the spinning process.

Factor (2) occasionally causes to more comber noil, more end-breakage and more drafting wave. This is clearly borne out by $R$. Bownass report ${ }^{[1]}$ that the percent reduction of mean fiber length on a worsted card reaches $20-65 \%$.

The fiber breakage is closely affected by the physical properties of fibers and their assemblies and by the mechanical actions of spinning process on the fiber material.
In other words, the variations of shape in the fiber length distribution are strongly affected by these properties and actions. Therefore, an analysis on variations in the fiber length distribution will give an aid to clarify the mechanical actions in the spinning operation.

In addition to $\mathrm{R}$. Bownass ${ }^{\text {[1] }}$ work, there are a number of works dealing with fiber breakage in spinning operations, e.g., D.A. Ross' ${ }^{[2]}$, P.P. Townend's ${ }^{[4,5]}$, R. Bownass' ${ }^{[3]}$ etc. They are all limited to experimental discussion on the relations between fiber breakage and the properties of materials. Byatt, et al's ${ }^{[6,7]}$ work seems uniquely interesting, in that it tries to find the equation to express variations in the fiber length distribution caused by fiber breakage, characterizing a breakage process by the breakage ratio and distribution of breaking points and also to solve the equation in the case of specific process, i. e., the breakage are occured proportionally to fiber length and uniformly along the fiber axis. 
Using the same concept as Byatt, et al., the present article discusses theoretically about the generalized breakage process and presents the results of applying this theory to experiments made on roller card.

\section{Equation on Fiber Breakage Process}

The auther makes an assumption that the breakage process is continuous and steady with operating time $t$, and that the loss of fibers during operating time may be ignored. Byatt, et al's concept can, therefore, be used here to deduce a breakage equation, by which to express the fiber length distribution at time $t$. To facilitate the theoritical treatment of process we replace the fiber length distribution with a number-frequency distribution of fibers. The results presented in this article are convertible into a weight-frequency distribution ${ }^{[n o t e 1]}$, as a matter of course.

We define the functions as follows:

$n(l, t) \Delta l$ : The expected number of fibers of length $l$ to $l+\Delta l$ included in the fibers of unit weight at time $t$.

$\alpha(l) \Delta t$ : The fraction of fibers of length $l$ that are broken in time $\Delta t$. Then, the reciprocal of $\alpha(l)$ means a life time. $\alpha(l)$ is referred to here as the "breakage ratio".

$\beta\left(l_{1} \rightarrow l\right) \Delta l$ : The fraction that the breakaging points are within $l$ to $l+\Delta l$ from one end of each fiber when fibers of length $l_{1}$, where $l<l_{1}$, are broken. $\beta\left(l_{1} \rightarrow l\right)$ is referred to here the "distribution of breaking points".

Take fibers $l$ to $l+\Delta l$ in length and consider the variations in amount of such fibers, during the interval $t$ to $t-\Delta t$, produced by:

(a) The decrease of fibers of length $l$ to $l+J l$ being broken into shorter fibers.

(b) The increase caused when the fibers of length $l_{1}$, where $l_{1}>l+\Delta l$, are broken into the fibers of length $l$ to $l+\Delta l$.

It is easy to see how the preceding item (a) takes the form of $-\alpha(l) n(l, t) \Delta t \Delta l$. As for item (b), since the number of fibers of $l$ to $\Delta l+l$ produced by the breakage of fibers of $l_{1}$ are $2 \alpha\left(l_{1}\right) \beta\left(l_{1} \rightarrow l\right) n(l, t) \Delta t \Delta l$, the item is obtained by integrating it from $l$ to $L$, the maximum length of fibers. Coefficient 2 is obtainable, assuming that $\beta\left(l_{1} \rightarrow l\right)$ is symmetrical function with respect to $l$.

Therefore, the following relation emerges:

$$
\begin{aligned}
& n(l, t+\Delta t) \Delta l-n(l, t) \Delta l=-\alpha(l) n(l, t) \Delta l \\
& +2 \int_{l}^{L} \alpha\left(l_{1}\right) \beta\left(l_{1} \rightarrow l\right) n\left(l_{1}, t\right) d l_{1} \Delta t \Delta l \quad \ldots . .
\end{aligned}
$$

By dividing both sides of the equation by $\Delta t \Delta l$ and making $\Delta t$ approach zero, we obtain the integro-differential equation, i.e.,

$$
\begin{aligned}
& \frac{\partial n(l, t)}{\partial t}=-\alpha(l) n(l, t) \\
& +? \int_{l}^{L} \alpha\left(l_{1}\right) \beta\left(l_{1} \rightarrow l\right) n(l, t) d l_{1}
\end{aligned}
$$

This equation will be hereinafter referred to as the "breakage equation".

We must remember that the definition of $n(l, t)$ means that $\int_{0}^{L} n(l, t) d l$ increases with time $t$ and agrees in the increase with the frequency of breakages occurred in unit weight of fibers in time $t$.

Consider the process in which such events as any one fiber breaks more than once can be ignored, defining the breakage ratio as the fraction of fibers broken throughout a process. Putting $t=0, \Delta t=1$ into e.q. (1), the breakage equation is transformed as follows:

$$
\begin{aligned}
& n(l, 1)=n(l, 0)-\alpha(l) n(l, 0) \\
& +2 \int_{l}^{L} \alpha\left(l_{1}\right) \beta\left(l_{1} \rightarrow l\right) n\left(l_{1}, 0\right) d l_{1}
\end{aligned}
$$

where $n(l, 0)$ is the initial distribution and $n(l, 1)$ is the final distribution. This process will be hereinafter referred to as the "single stage breakage process".

\section{Solving the Brəakage Equation}

Now, slove eq. (2) for $n(l, t)$ under the initial condition of distribution. By applying the Laplace transformation to eq. (2), the breakage equation is transformed to following eq. (5), whitch is Volterra's linear integral equation of the second kind having kernel $K$ expressed by

$$
K\left(l, l_{1}, s\right)=\frac{\alpha\left(l_{1}\right) \beta\left(l_{1} \rightarrow l\right)}{s+\alpha(l)}
$$

which is

$$
\begin{aligned}
& n(l, s)=\frac{n(l, 0)}{s+\alpha(l)} \\
& +2 \int_{l}^{L} \alpha\left(l_{1}\right) \beta\left(l_{1} \rightarrow l\right) \frac{n\left(l_{1}, s\right)}{s+\alpha(l)} d l_{1}
\end{aligned}
$$

where, $n(l, s)$ is Laplace transform of $n(l, \mathrm{t})$.

To solve the eq. (5) is comparatively easy in the case of a specific kernal and a little complex in the general cases. Even if the solution is found, its inverse transform is rather complex. For this, we consider simple process and then, deal with the general process.

3-1. Where $\alpha(l)=k l^{n}, \beta\left(l_{1} \rightarrow l\right)=1 / l_{1}$

The expression for $\alpha(l)$ indicates that longer fibers are the breakage-prone, and for $\beta\left(l_{1} \rightarrow l\right)$ indicates that breakage occurs evenly along the fiber axis. The solution of this process is the basis of later theoretical evolution, as we shall see.

Differentiating eq. (5) with respect to $l$, results in:

$$
\frac{\partial n(l, s)}{\partial l}+\frac{k(n+2) l^{n-1}}{s+k l^{n}} n(l, s)=\frac{\partial n(l, 0)}{\partial l} \cdots(6)
$$

By applying the inverse Laplace transformation to the solution of above differential equation, we obtain,

$$
\begin{aligned}
& n(l, t)=e^{-k l^{n} t} n(l, 0) \\
& +2 k \int_{l}^{L} l_{1}{ }^{n-1} g\left(l, l_{1}, t\right) n\left(l_{1}, 0\right) d l_{1}
\end{aligned}
$$


where $g$ is

$$
g\left(l, l_{1}, t\right)=L^{-1} \frac{\left(s+k l_{1}^{n}\right)^{\frac{2}{n}-1}}{\left(s+k l^{n}\right)^{\frac{2}{n}+1}}
$$

It is difficult to calculate eq. (8) about artbitary $n$ directly, however, it is possible to obtain with series form solution by expanding the image function with respect to $s$. The solution by this method is included in the solution made in $3-2$, as a matter of cause. Therefore, we discuss only the simpler cases in this section, assuming $m=2 / n$ where $m$ is a positive integer. In this case, eq. (8) transforms thus:

$$
g\left(l, l_{1}, t\right)=\frac{1}{m !} \lim _{s \rightarrow k l^{n}} \frac{d^{m}}{d s^{m}}\left(s+k l_{1}^{n}\right)^{m-1} e^{s t}
$$

As the examples:

(i) Assuming $n=2$,

$$
n(l, t)=e^{-k l^{2} t}\left[n(l, 0)+2 k t \int_{\ell}^{L} l_{1}, n\left(l_{1}, 0\right) d l_{1}\right]
$$

(ii) and assuming $n=1$,

$$
\begin{aligned}
n(l, t)= & e^{-k l t}\left[n(l, 0)+2 k t \int_{l}^{L} n\left(l_{1}, 0\right) d l_{1}\right. \\
& \left.+k^{2} t^{2} \int_{l}^{L}\left(l_{1}-l\right) n\left(l_{1}, 0\right) d l_{1}\right] \ldots .
\end{aligned}
$$

\section{3-2 General Processes}

The two fractions, i.e., $\alpha(l)$ and $\beta\left(l_{1} \rightarrow l\right)$, in the everyday process are not so easily obtainable as preceding section 3-1 suggests. We, therefore, discuss a solution in a case where these fractions are generally given.

Assuming the solution of breakage equation to be,

$$
n(l, s)=\sum_{n=0}^{\infty} 2^{n} \emptyset_{n}(l, s)
$$

and substituting this into eq. (5), we find that $\Phi_{n}$ ought to satisfy the following relations,

$$
\begin{aligned}
& \emptyset_{0}(l, s)=\frac{n(l, 0)}{s+\alpha(l)} \\
& \emptyset_{n}(l, s)=\int_{l}^{I} K\left(l, l_{1}, s\right) \emptyset_{n-1}\left(l_{1}, s\right) d l_{1}
\end{aligned}
$$

With the integral operater $A$ defined as,

$$
\Phi_{1}(l, s)=A \emptyset_{0}(l, s)=\int_{l}^{I} K\left(l, l_{1}, s\right) \emptyset_{0}\left(l_{1}, s\right) d l_{1}
$$

eq. (12) can be presented by following series,

$$
n(l, s)=\emptyset_{0}(l, s)+\sum_{n=1}^{\infty} 2^{n} A^{n} \emptyset_{0}(l, s)
$$

Transforming this into time domain, the solution is obtained as

$$
n(l, t)=e^{-\alpha(l) t} n(l, 0)+\sum_{n=1}^{\infty} 2^{n} B^{n} \phi_{n}
$$

where $B^{n} \varphi_{n}$ is

$$
\beta^{n} \phi_{n}=L^{-1} A^{n} \emptyset_{0}(l, s)
$$

$\varphi_{n}$ is

$$
\phi_{n}=L^{-1} \frac{n\left(l_{n}\right) ।}{s+\alpha(l)} \prod_{i=1}^{n} \frac{1}{s+\alpha\left(l_{i}\right)}
$$

and the operater $B$ is defined as

$$
\begin{aligned}
& B \phi_{1}=\int_{l}^{l .} \alpha\left(l_{1}\right) \beta\left(l_{1} \rightarrow l\right) \phi_{1} d l_{1} \\
& B^{n} \phi_{n}=\int_{l}^{L} \alpha\left(l_{1}\right) \beta\left(l_{1} \rightarrow l\right) \int_{l_{1}}^{I .} \ldots \ldots \ldots \ldots . . \\
& \ldots \ldots \ldots . \int_{l_{n-1}}^{L} \alpha\left(l_{n}\right) \beta\left(l_{n} \rightarrow l_{n-1}\right) \phi_{n} d l_{n} \ldots \ldots d l_{1}
\end{aligned}
$$

The calculation of eq. (18) is easy, however, merely substituting it into eq. (16) is not realistic. For this reason, assuming $\alpha(l) t$ to be small and expanding eq. (18) into the power series of $\alpha\left(l_{i}\right) t$ lead to a concrete solution of eq. (16), as follow:

$$
\begin{aligned}
& n(l, t) \\
& =e^{-\alpha(l) t}\left[n(l, 0)+2 t \int_{l}^{L} \alpha\left(l_{1}\right) \beta\left(l_{1} \rightarrow l\right) n\left(l_{1}, 0\right) d l_{1}\right. \\
& +\frac{t^{2}}{2 !}\left\{2 \int_{l}^{L} \alpha\left(l_{1}\right) \beta\left(l_{1} \rightarrow l\right)\left\{\alpha(l)-\alpha\left(l_{1}\right)\right\} n\left(l_{1}, 0\right) d l_{1}\right. \\
& +2^{2} \int_{l}^{L} \alpha\left(l_{1}\right) \beta\left(l_{1} \rightarrow l\right) \int_{l_{1}}^{L} \alpha\left(l_{2}\right) \beta\left(l_{2} \rightarrow l_{1}\right) n\left(l_{2}, 0\right) \\
& \left.\left.d l_{2} d l_{1}\right\}+\cdots \cdots\right] \quad \cdots \quad(20)
\end{aligned}
$$

Assuming $\alpha(l) t$ to be small is considered reasonable for the purpose of this article, in that it deals with breakage in the spinning process.

Eq. (20) is identical with the solution on the process discussed in 3-1. This is easily brone out by calculating the terms of eq. (20), respectively. The first term of eq. (20) is identical with the solution obtained if the second term of the right-hand side of eq. (2) is zero. Clearly, then, it indicates the number of fibers unbroken after time $t$. Accordingly, the second and subsequent terms express the number of fibers generated by breaking.

Further expanding eq. (20) with respect to $\alpha(l) t$, the following is obtainable:

$$
n(l, t)=n(l, 0)-t I_{1}(l)+\frac{t^{2}}{2 !} I_{2}(l)-\frac{t^{3}}{3 !} I_{3}(l)+\cdots
$$


where $I_{i}(l)$ is

$$
\begin{aligned}
& I_{1}(l)=\alpha(l) n(l, 0)-2 \int_{l}^{L} \alpha\left(l_{1}\right) \beta\left(l_{1} \rightarrow l\right) n\left(l_{1}, 0\right) d l_{1} \\
& I_{i}(l)=\alpha(l) I_{i-1}(l)-2 \int_{l}^{L} \alpha\left(l_{1}\right) \beta\left(l_{1} \rightarrow l\right) I_{i-1}\left(l_{1}\right) d l_{1}
\end{aligned}
$$

Ii $(l)$ shows the final distribution, if the single stage breakage process with initial distribution $I_{i-1}(l)$ is taken into account. Thus, the solution, when approximated to the second terms, agrees with the single stage breakage process.

If the integration of the right-hand side of the breakage equation is possible, the existance of a solution and convergences of eq. (20) and eq. (21), are obvious. It is also obvious that the smaller values of $\alpha(l) t$ (especially smaller degree of dependence of $\alpha(l)$ upon $l$ ) leads to the faster convergence.

\section{Variation in Moment of Distribution}

The fiber length distribution in a arbitrary time is the process where the two fractions are obtainable by calculating eq. (20) or (21). In many cases, however, we want to find two fractions, i.e., $\alpha(l)$ and $\beta\left(l_{1} \rightarrow l\right)$, when the initial and final distribution are already known.

A conceivable method to do so is to consider that the moment of the final distribution is strongly influenced by two fractions. By this method, Meyer et. al. ${ }^{[9]}$ discusses a case where the breakage ratio is proportional to the fiber length and presents successful results.

The m-th moment denoted by $M_{m}(t)$ in time $t$ is expressed from the difinition of the moment, as follows:

$$
M_{m}(t)=\frac{1}{N(t)} \int_{0}^{I \cdot} l^{m} n(l, t) d l
$$

Substituting eq. (21) into eq. (23), the follwing is obtainable:

$$
\begin{aligned}
M_{m}(t) N(t) & =M_{m}(0) N(0)-t \int_{0}^{L} l^{m} I_{1}(l) d l \\
& +\frac{t^{2}}{2 !} \int_{0}^{L} l^{m} I_{2}(l) d l-\cdots . .
\end{aligned}
$$

where $N(t)$ is zero-th moment, i.e., the total number of fibers in a unit weight.

Now, let $\beta_{0}(x),(0 \leq x \leq 1)$, to be the distribution of breaking points of fibers of unit length. Then, since

$$
\beta\left(l_{1} \rightarrow l\right)=\frac{1}{l_{1}} \beta\left(\frac{l}{l_{1}}\right)
$$

and from this

$$
\begin{aligned}
& \int_{0}^{L} l^{m} \int_{l}^{I} \alpha^{n}(l) \beta\left(l_{1} \rightarrow l\right) I_{i}\left(l_{1}\right) d l_{1} d l \\
& =\int_{0}^{I} l^{m} \alpha^{n}(l) I_{i}(l) d l \int_{0}^{1} x^{m} \beta_{0}(x) d x
\end{aligned}
$$

eq. (24) is reduced as follows:

$$
\begin{aligned}
& M_{m}(t) N(t)=M_{m}(0) N(0) \\
& \quad+\beta_{m}\left\lceil t \int_{0}^{L} l^{m} \alpha(l) n(l, 0) d l\right. \\
& \left.\quad-\frac{t^{2}}{2 !} \int_{0}^{L} l^{m} \alpha(l) I_{2}(l) d l+\cdots \cdots\right]
\end{aligned}
$$

where

$$
\beta_{m}=2 \int_{0}^{1} x^{m} \beta_{0}(x) d x-1
$$

If $m \geq 0, \beta_{m}$ for arbitrary $\beta_{0}(x)$ takes the value of -1 to 1 .

(i) Where $m=1$

If $m=1$, then $\beta_{m}=0$. Therefore, the mean fiber length $l(t)$ is easily yielded and is

$$
\bar{l}(t)=\frac{N(0)}{N(t)} \bar{l}(0)
$$

This is a natural result, because the loss of fibers is ignored in this discussion.

(ii) Where $m=0$.

If $m=0$, then $\beta_{m}=1$. Therefore, the total number of fibers in unit weight at time $t$ is

$$
\begin{aligned}
& N(t)=N(0)\left[1+t \int_{0}^{L} \alpha(l) n(l, 0) d l\right. \\
& -\frac{t^{2}}{2 !} \int_{0}^{L} \alpha(l) I_{1}(l) d l+\frac{t^{3}}{3 !} \int_{0}^{L} \alpha(l) I_{2}(l) d l \\
& -\cdots \cdots] \quad \ldots \ldots \ldots \ldots . .
\end{aligned}
$$

Defining $M$ as

$$
M=\max \alpha(l)
$$

the upper bound of $N(t)$ is expressed as following:

$$
\begin{aligned}
N(t) & \leqq N(0)\left[1+t M+\frac{t^{2}}{2 !} M^{2}+\cdots \cdots\right] \\
& =N(0) e^{t M}
\end{aligned}
$$

The equality symbol is valid if the breakage ratio is independent of $l$.

5. The Moment where $\alpha(l)=k l^{n}$

Assuming the breakage ratio is proportional to $l^{n}$, i.e., $\alpha(l)=k l^{n}$, which agrees comperatively with experimental results and using the results cited in the previous chapter, we discuss about the distribution of breaking points. Substituting $k l^{n}$ into eq. (27), the following is obtainable:

$$
\begin{aligned}
& M_{m}(t) N(t)=N(0)\left[M_{m}(0)\right. \\
& +t k \beta_{m} M_{n+m}(0)+\frac{t^{2} k^{2}}{2 !} \beta_{m} \beta_{m}+{ }_{n} M_{m+2 n}(0) \\
& \left.+\frac{t^{3} k^{3}}{3 !} \beta_{m} \beta_{m+n} \beta_{m+2 n} M_{m+3 n}(0)+\cdots \cdots\right]
\end{aligned}
$$


This shows that moment after time $t$ is experssible by the sum of products of the moment of the initial distribution and the moment of distribution of breaking points.

Replacing $\beta_{0}(x)$ with Pearson's (Type I) distribution which agrees in tendency with expeimental results that the breaking points are apt to be generated around the center of fiber, we discuss the characteristic of $\beta_{m}$. Paerson's distribution is expressed by following:

$$
\beta_{0}(x)=c\{x \quad(1-x)\}^{J}
$$

Fig. 1 shows $\beta_{0}(x)$ at $J=0,1,2$, and 3. Assuming $J=0$, then $\beta_{0}(x)=1$. This may be called "random breakage".

Fig. 2 shows $\beta_{m}$ as a function of $m$ for these distributions. It is clear the more breakage-prone around the center are the higher the gradual speed toward -1 . The dotted lines in Figs. 1 and 2 are not very realistic, but are intended to illustrate the proneness to breakage of fibers at leading ends. In this case, the function is given by

$$
\beta_{0}(x)=3(2 x-1)
$$

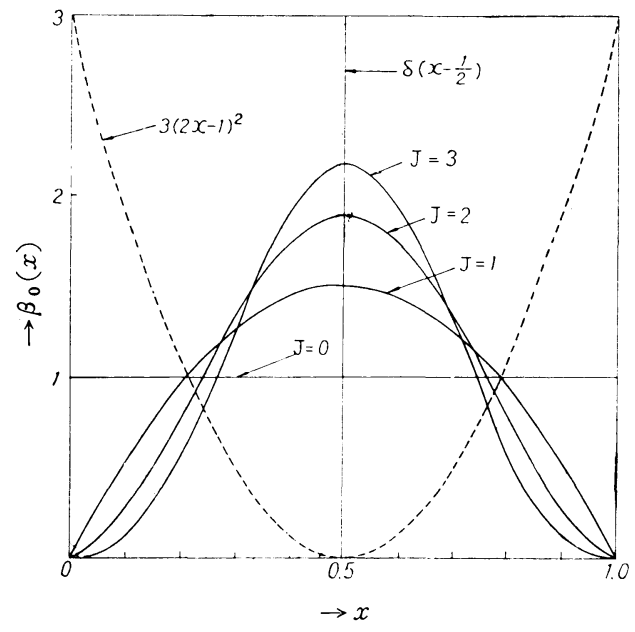

Fig.1 Examples of various distributions of breaking points

The evidence so far indicates that the lower the convergence velocity of moment of eq. (33) is, the more breakage-prone fibers away from the center are.

Assuming $m=0$ in eq. (33), the approximation up to the second term is obtainable as

$$
\frac{N(t)}{N(0)}=1+t k M_{n}(0)+\frac{t^{2} k^{2}}{2 !} \beta_{n} M_{2 n}(0) \cdots
$$

With this relation, it is possible to estimate approximate $\beta_{n}$ if the breakage ratio is known; and $k$, if $n$ and $\beta n$ are known.

\section{Results of Experiments}

Here are the results of appling the foregoing theory to experiments made on roller card.

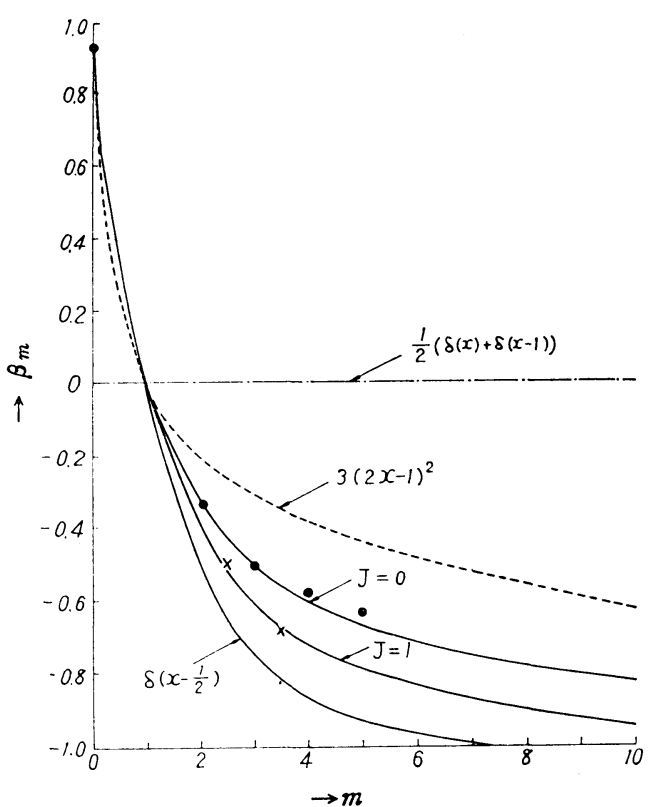

Fig. $2 \beta \mathrm{m}$ in various distributions of breaking points

\section{6-1. Method of Experiments}

The used fibers were 1.5 den, regular viscose staple with nominal length each 120, 90 and $50 \mathrm{~mm}$. The sample fibers were adequately opened manually in advance and then carded repeatedly. The purpose of pre-opening was to prevent the steady state in the process from being disturbed by the differences in the degree of opening during different rounds of carding.

The fiber length was measured sampling 1,000 to 2,000 fibers from the same sample and measuring them with a rule in unit of $\mathrm{mm}$.

\section{6-2. Breakage Ratio}

The experimental fiber length distributions are given in Figs. 5 to 9 . Where $n_{0}(l, t)$ is defined as

$$
n_{0}(l, t)=\frac{1}{N(t)} n(l, t)
$$

It is clear from the figures that the longer fibers, more breakage-prone, therefore we assume again $\alpha(l)=k l^{n}$.

One conceivable way to obtain the breakage ratio is to use the following equation to express the number of fibers unbroken even after the process, as

$$
n(l, t)=e^{-\alpha^{\prime}(l) t} n(l, 0)
$$

The fibers to be near the nominal length are not only the unbroken fibers, but include the broken fibers having the breakage points near the leading end of original fibers. For this reasons, it is required to find a proper method to distinguish experimentally either being the broken fibers or not. One of the methods considered is 
to use the sample fibers having been dyed both leading ends by some fluoresent dystuff. In this article, however, the calculation of breakage ratio was carried out by using the approximate estimation of the unbroken fibers.

As shown in Fig. 3 , if we take $l_{1}$ in such a way as to make $L-l_{1}$ to sufficently small compared with $L$, and then look at the fibers exceeding $l_{1}$ in length, we shall find that breakage ratio conforms approximately to $k l$. And we assume that $\beta_{0}(x)=1$.

From the assumtions, eq. (11) is reduced to following to express the number of fibers exceeding $l_{1}$ :

$$
\int_{0}^{L} n(l, t) d l=N_{1}(t)
$$

where $N_{1}(t)$ is defined as

$$
N_{1}(t)=N_{1}(0) e^{-k t l_{1}}\left[1+k t \bar{l}_{1}(0)-k t l_{1}\right] \cdots
$$

which, by the 2 nd order approximation with respect to $k t$ transforms into:

$$
\begin{aligned}
N_{1}(t) & =N_{1}(0)\left[1+k t\left\{\bar{l}_{1}(0)-2 l_{1}\right\}\right. \\
& \left.-k^{2} t^{2} l_{1}\left\{\bar{l}_{1}(0)-\frac{3 l_{1}}{2}\right\}\right]
\end{aligned}
$$

by which it is possible to obtain $k t$. Here $\overline{l_{1}}(0)$ is the initial mean of lengths exceeding $l_{1}$. This method is believed to have high-degree precision for a initial distribution with the small variance of lengths such as is used in this experiment.

Table 1-A shows the results of calculation by eq. (41). Here, we defined that $t$ agrees with the number of carding rounds when $t$ takes an integer. The correlation between the variations of estimated $k$ within the same nominal length and the frequency of carding is hardly noticeable. This shows that the manual pre-opening to keep the steady state of the process was meaningful. If the breakage ratio is proportional to $l$, the value of $k$ ought to be invariable between different nominal lengths. But the results of Table 1-A show that the longer the nominal length, the higher the value. This means $n>1$.

The mark $x$ in Fig. 4 shows the logarithms plotted for $\overline{k l_{1}}(0)$ and $\overline{l_{1}}(0)$. Since these points are well along the linear line, the breakage ratio seems to satisfy $k l^{n}$ as far as our experiments are concerned. We obtained $n=2.50$ to calculate the gradient. The forgoing is a case where $\beta_{0}(x)=1$. It is clear from Figs. 5 to 9 that the breaking points concentrate around the center of fiber axis. Therefore. the above results seem to overestimate the breakage ratio.

Bearing this in mind and assuming the absence of fibers of length $l_{1}$ to $l_{1}-L$ which, after breakage, are still similar in length to this range, eq. (38) may be used. Approximating eq. (38) to 2 nd order and putting $l_{1}$ into $l$, the following is obtainable:

$$
N_{1}(t)=N_{1}(0)\left[1-k t \bar{l}_{1}(0)+\frac{\left.k^{2} t^{2} \bar{l}_{1}^{2}(0)\right] \cdots}{2}\right.
$$

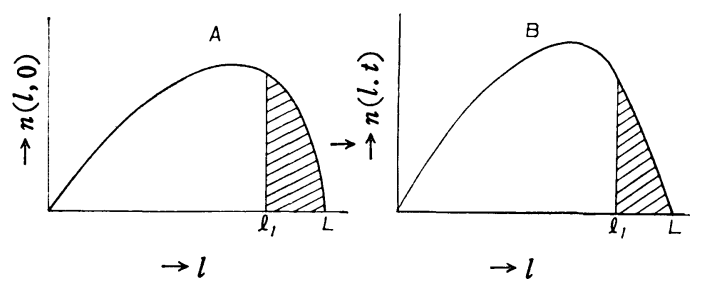

Fig.3 Illustrations of calculations of breakage ratios

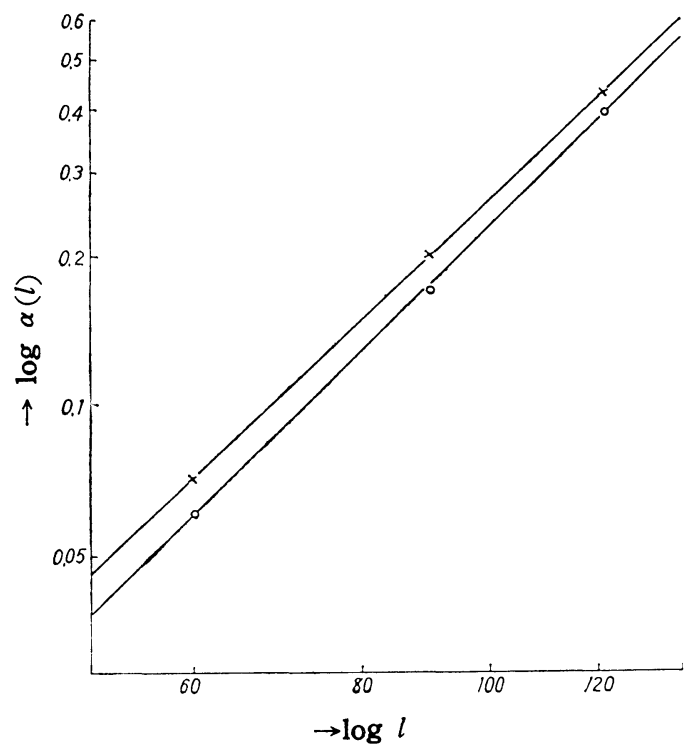

Fig.4 Relation between breakage ratio and and fiber length

where $\overline{l^{2}}(0)$ is the initial 2 nd moment exceeding $l_{1}$.

The value $k$ obtained from this relation is shown by the mark $\bigcirc$ in Fig. 4 and Table 1-B. Naturally, $k$ is smaller than if eq. (41) is used and is 2.57 .

After all, the breakage ratio is proportional to $l^{2.5-2.6}$ Consequently, the following equation will be used hereinafter for calculation:

$$
\alpha(l)=k l^{2 \cdot 5}
$$

$k$ in this case should be $2.43 \times 10^{--6}$, averaging the value from two calculation methods.

6-3. Distribution of Breakaing Points

Assuming eq. (43) to be breaking ratio, $\beta_{m}$ calculated from eq. (36) and (20) is plotted by mark $\times$ in Fig. 2 . They are almost on the line $J=1$. And mark shows $\beta_{m}$ calculated by the first order approximation of eq. (33). These plots are almost on the line $J=0$. The former estimate for $m \beta$ is smaller than latter, which is clarified to consider the second term being positive when $m>1$. Consequently, we will use the following hereinafter:

$$
\beta_{0}(x)=6 x(1-x)
$$


Table 1 Estimated vaues of Breakage ratio

\begin{tabular}{|c|c|c|c|c|c|c|c|}
\hline $\begin{array}{c}\text { Cut :length } \\
(\mathrm{mm})\end{array}$ & & $0 \sim 1$ round & $1 \sim 2$ rounds & $2 \sim 3$ rounds & Average & $n=2.5$ & $n=2.6$ \\
\hline 60 & $\begin{array}{c}k \bar{l}_{1}(0) \\
k\end{array}$ & $\begin{array}{l}0.059 \\
0.099 \times 10^{-2}\end{array}$ & $\begin{array}{l}0.095 \\
0.158^{\times 10^{-2}}\end{array}$ & $\begin{array}{l}0.058 \\
0.097^{\times 10^{-2}}\end{array}$ & $\begin{array}{l}0.071 \\
0.118^{\times 10^{-2}}\end{array}$ & $2.55^{-} \times 10^{-6}$ & $1.69^{-} \times 10^{-6}$ \\
\hline 90 & $\begin{array}{c}k \bar{l}_{1}(0) \\
k\end{array}$ & $\begin{array}{l}0.224 \\
0.248^{\times 10^{-2}}\end{array}$ & $\begin{array}{l}0.206 \\
0.228^{\times 10^{-2}}\end{array}$ & $\begin{array}{l}0.172 \\
0.191 \times 10^{-2}\end{array}$ & $\begin{array}{l}0.200 \\
0.222^{\times 10^{-2}}\end{array}$ & $2.59^{-} \times 10^{-6}$ & $1.65^{\times 10^{-6}}$ \\
\hline 120 & $\begin{array}{c}k \bar{l}_{1}(0) \\
k\end{array}$ & $\begin{array}{l}0.406 \\
0.334^{\times 10^{-2}}\end{array}$ & $\begin{array}{l}0.431 \\
0.354^{\times 10^{-2}}\end{array}$ & $\begin{array}{l}0.416 \\
0.342^{\times 10^{-2}}\end{array}$ & $\begin{array}{l}0.417 \\
0.343^{\times 10^{-2}}\end{array}$ & $2.55^{-} \times 10^{-6}$ & $1.58^{-} \times 10^{-6}$ \\
\hline & & & & & Average & $2.56^{\times 10^{-6}}$ & $1.64^{\times 10^{-6}}$ \\
\hline
\end{tabular}

B

\begin{tabular}{|c|c|c|c|c|c|c|c|}
\hline 60 & $\begin{array}{c}k \bar{l}_{1}(0) \\
k\end{array}$ & $\begin{array}{l}0.052 \\
0.086^{\times 10^{-2}}\end{array}$ & $\begin{array}{l}0.079 \\
0.131 \times 10^{-2}\end{array}$ & $\begin{array}{l}0.049 \\
0.082 \times 10^{-2}\end{array}$ & $\begin{array}{l}0.060 \\
0.100^{\times 10^{-2}}\end{array}$ & $2.15^{-} \times 10^{-6}$ & 1. $43^{\times 10^{-6}}$ \\
\hline 90 & $\begin{array}{c}k \bar{l}_{1}(0) \\
k\end{array}$ & $\begin{array}{l}0.200 \\
0.222^{\times 10^{-2}}\end{array}$ & $\begin{array}{l}0.186 \\
0.206^{\times 10^{-2}}\end{array}$ & $\begin{array}{l}0.153 \\
0.170^{\times 10^{-2}}\end{array}$ & $\begin{array}{l}0.180 \\
0.199 \times 10^{-2}\end{array}$ & $2.33^{-} \times 10^{-6}$ & $\frac{-}{1.48^{\times 10^{-6}}}$ \\
\hline 120 & $\begin{array}{c}k \bar{l}_{1}(0) \\
k\end{array}$ & $\begin{array}{l}0.392 \\
0.322^{\times 10^{-2}}\end{array}$ & $\begin{array}{l}0.403 \\
0.331 \times 10^{-2}\end{array}$ & $\begin{array}{l}0.381 \\
0.313^{\times 10^{-2}}\end{array}$ & $\begin{array}{l}0.392 \\
0.322^{\times 10^{-2}}\end{array}$ & $2.41^{-} \times 10^{-6}$ & $1.49^{--}$ \\
\hline & & & & & Average & $2.30^{\times 10^{-6}}$ & $1.47^{\times 10^{-6}}$ \\
\hline
\end{tabular}

\section{6-4. Calculated Distribution after Carding}

Figs. 5 to 9 show, with tangent lines, results obtained by numerical calculation of eq. (21) where both functions are given by eq. (43) and (44) and a sample before carding is taken as the initial distribution. The histogram shown by dotted lines is the initial distribution and solid line shows the mesaured value after carding. Table 2 compares measured values with calculated values of total number $N(t)$, average length $\overline{l(t)}$ and average length $\overline{l w(t)}$ on the weight-frequency distribution.

The calculatecd values agree generally with measured values. But, comparing calculated with measured distribution, we can not overlooked the fact that there are discrepancies having following two common tendencies:

a) The calculated value for extremly short fibers is high. This is mainly because, the shorter the fiber, the more lossprone it is, during carding and sampling.

b) The calculated value near a quarter of a nominal length is low and the one near three quarter is high. This means that the number of fibers having been broken down more than once, are more than the number of fibers expected by the theory. It is difficult to explain this fact reasonablly, bearing in mind P.P. Townend's findings that, in the blending of long and short fibers, more long fibers break than when long fibers alone are used.

If $J=2$, comparing with result when $J=1$, the discrepancy (a) is generally small but the over all agreement is decrease.

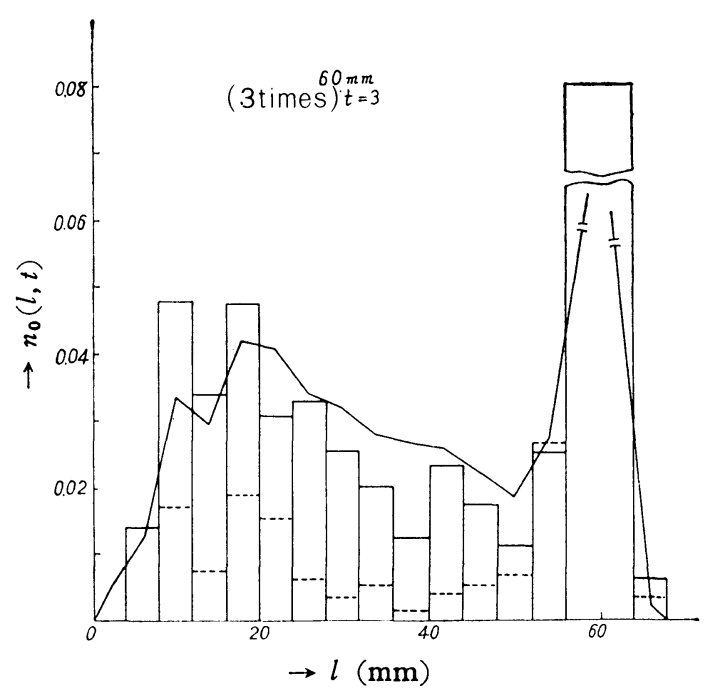

Fig.5 Experimental distribution of fiber lengths where nominal length is $60 \mathrm{~mm}$ and frequency of carding $\mathrm{t}=3$.

\section{The Process with Loss of Fibers}

Theories described as far ignore the loss of fibers. It is clear from the results of our experiments, however, we must take the fiber loss in account to express more precisely the breakage process. For this, consider the process with loss of fibers. 


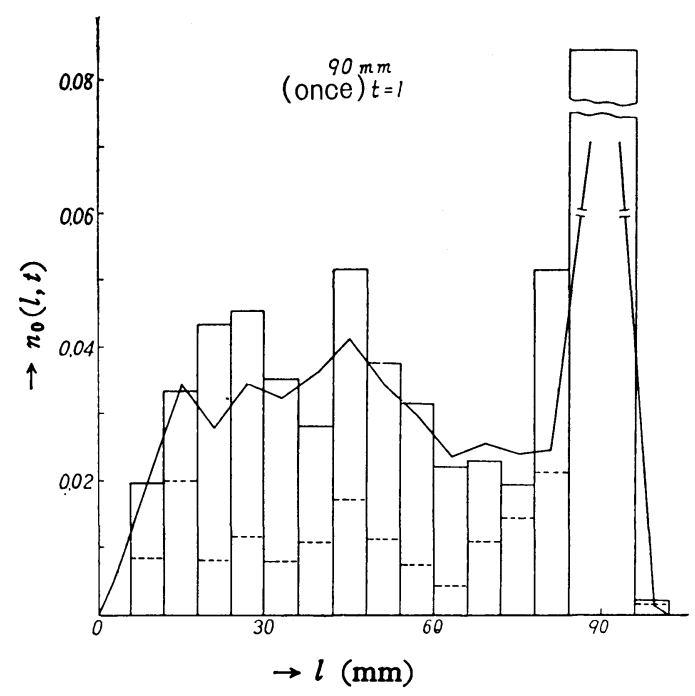

Fig.6 Experimental distribution of fiber lengths, where nominal length is $90 \mathrm{~mm}$ and frequency of carding $\mathrm{t}=1$

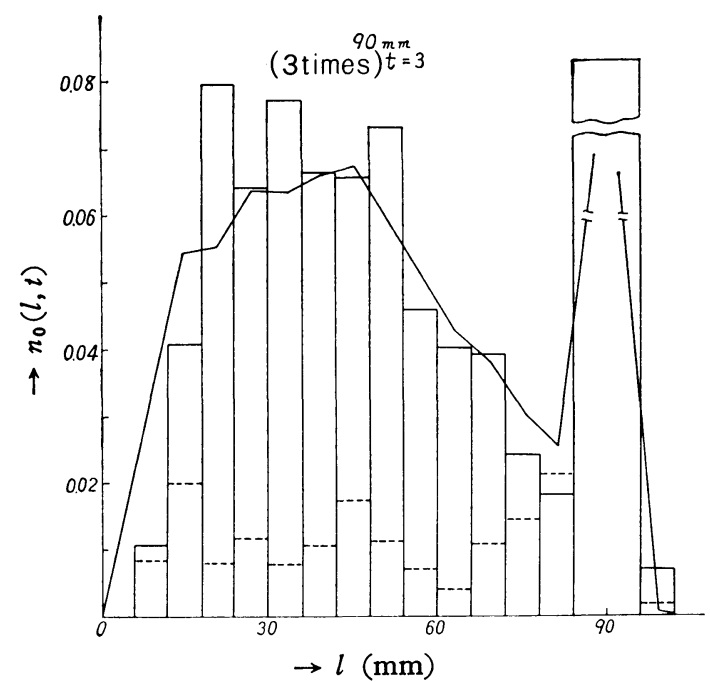

Fig.7 Experimental distribution of fiber lengths, where nominal length is $120 \mathrm{~mm}$ and frequency of carding $\mathrm{t}=1$

Here, the loss ratio $\gamma(l)$ is defined as follow:

$\gamma(l) t$ : The fraction of fibers of length $l$ that are lost in time $\Delta t$.

It is reasonable that this is a decreasing function with $l$.

Using this function, the following equation carresponding to breakage equation (2) is obtainable:

$$
\begin{aligned}
\frac{\partial n(l, t)}{\partial t} & =-\{\alpha(l)+\gamma(l)\} n(l, t) \\
& +2 \int_{l}^{L} \alpha\left(l_{1}\right) \beta\left(l_{1} \rightarrow l\right) n\left(l_{1}, t\right) d l_{1} \ldots \text { (45) }
\end{aligned}
$$

which, when solved, makes a solution coresponding to

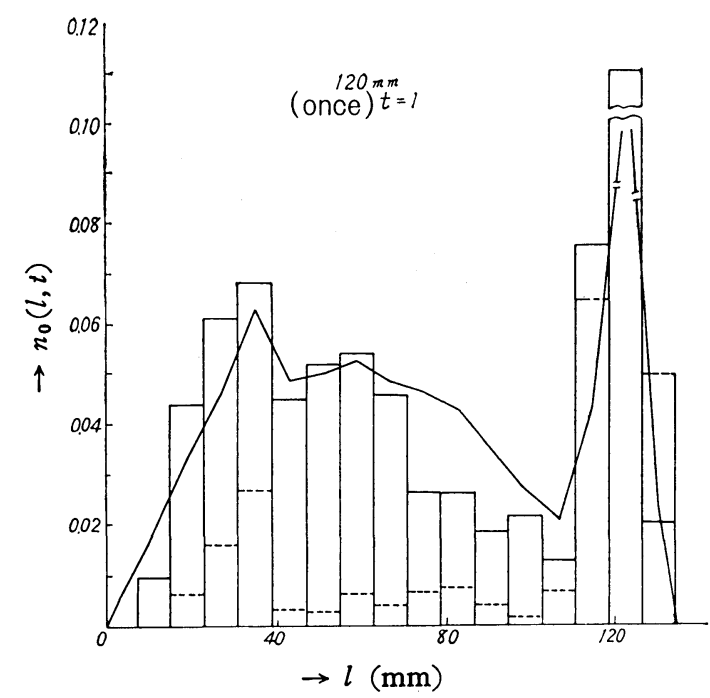

Fig.8 Experimental distribution of fiber lengths, where nominal length is $90 \mathrm{~mm}$ and frequency of carding $\mathrm{t}=3$

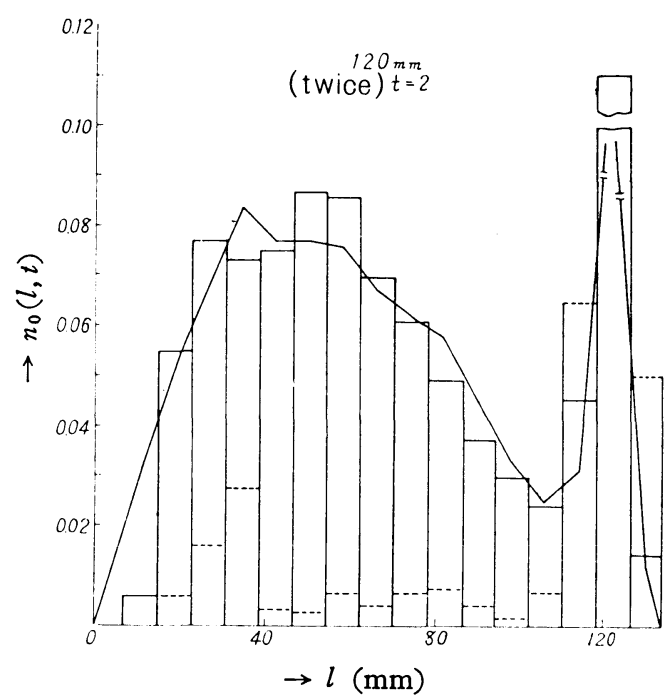

Fig.9 Experimental distribution of fiber lengths, where nominal lengths is $120 \mathrm{~mm}$ and frequency of carding $\mathrm{t}=\mathbf{2}$

eq. (20), thus:

$$
\begin{aligned}
& n(l, l)=e^{-\varepsilon\langle l\rangle t}[n(l, 0) \\
& +2 t \int_{l}^{L} \alpha\left(l_{1}\right) \beta\left(l_{1} \rightarrow l\right) n\left(l_{1}, 0\right) d l_{1} \\
& +\frac{t^{2}}{2 !}\left\{2 \int_{l}^{L} \alpha\left(l_{1}\right) \beta\left(l_{1} \rightarrow l\right)\left\{\varepsilon(l)-\varepsilon\left(l_{1}\right)\right\} n\left(l_{1}, 0\right) d l_{1}\right. \\
& +2^{2} \int_{l}^{L} \alpha\left(l_{1}\right) \beta\left(l_{1} \rightarrow l\right) \int_{l_{1}}^{L} \alpha\left(l_{2}\right) \beta\left(l_{2} \rightarrow l_{1}\right) n\left(l_{2}, 0\right) \\
& \left.\left.d l_{2} d l_{1}\right\}+\cdots \cdots\right] \quad \ldots \quad(46)
\end{aligned}
$$

where $\varepsilon(l)$ is 
Table 2 Comparing measured values with calculated values of total nnmber $N(t)$, average lenght $\bar{l}(t)$ and average length $\vec{l} w(t)$ on the weight -pequency distribution

\begin{tabular}{|c|c|c|c|c|c|c|c|c|}
\hline \multirow{2}{*}{$\begin{array}{l}\text { Cut } \\
\text { length } \\
\text { (mm) }\end{array}$} & \multirow{2}{*}{$\begin{array}{l}\text { Average } \\
\text { length }(\mathrm{mm})\end{array}$} & \multirow{2}{*}{\begin{tabular}{l}
\multicolumn{1}{l}{$\left.\begin{array}{l}\text { initial } \\
(t=0\end{array}\right)$} \\
$\begin{array}{l}\text { Measured } \\
\text { value }\end{array}$
\end{tabular}} & \multicolumn{2}{|c|}{$1 \mathrm{st}(t=1)$} & \multicolumn{2}{|c|}{$2 \mathrm{nd}(t=2)$} & \multicolumn{2}{|c|}{$3 \mathrm{rd}(t=3)$} \\
\hline & & & $\begin{array}{l}\text { Measured } \\
\text { value }\end{array}$ & \begin{tabular}{|l|} 
Cal culated \\
value
\end{tabular} & $\begin{array}{l}\text { Measured } \\
\text { value }\end{array}$ & $\begin{array}{l}\text { Calculated } \\
\text { value }\end{array}$ & $\begin{array}{l}\text { Measured } \\
\text { value }\end{array}$ & \begin{tabular}{|l}
$\begin{array}{l}\text { Calculated } \\
\text { value }\end{array}$ \\
\end{tabular} \\
\hline \multirow{3}{*}{60} & $N(t)$ & 1.000 & 1.036 & 1.061 & 1.055 & 1.119 & 1.155 & 1.176 \\
\hline & $\bar{l}(t)$ & 56.3 & 54.4 & 53.1 & 53.4 & 50.3 & 48.8 & 47.9 \\
\hline & $\bar{l}_{w}(t)$ & 58.6 & 58.0 & 57.1 & 56.8 & 55.7 & 55.3 & 54.3 \\
\hline \multirow{3}{*}{90} & $N(t)$ & 1.000 & 1.194 & 1.160 & 1.369 & 1.306 & 1.401 & 1.441 \\
\hline & $\bar{l}(t)$ & 83.6 & 70.0 & 72.1 & 61.0 & 64.0 & 59.7 & 58.1 \\
\hline & $\bar{l}_{w}(t)$ & 87.5 & 80.1 & 81.5 & 74.7 & 76.3 & 72.2 & 71.7 \\
\hline \multirow{3}{*}{120} & $N(t)$ & 1.000 & 1.338 & 1.342 & 1.644 & 1.632 & & \\
\hline & $\bar{l}(t)$ & 116.9 & 87.4 & 87.1 & 71.1 & 71.7 & & \\
\hline & $\bar{l}_{w}(t)$ & 120.7 & 104.5 & 103.4 & 91.8 & 91.5 & & \\
\hline
\end{tabular}

$$
\varepsilon(l)=\alpha(l)+\gamma(l)
$$

Further treatment of this is reserved for inquring at a later date.

\section{Acknowlegdments}

The auther thanks prof. S. Ishikawa and Associate prof. J. Shimijzu of the Tokyo Institute of Technology, and Prof. T. Ogawa of Yamagata University for their guidance in our research. Special thanks are due to Mr. Y. Nakajima, Fujii Keori KK for his assistance in our experiments.

\section{Literature cited}

[1] R. Bownass; Wool Text. Res. Conf. Austria, E172-203 (1955)

[2] D.A. Ross, R.L. Bratt, L.F. Story; J. Text. Inst., 51 T907-921 (1960)

[3] R. Bownass; J. Text. Inst., 51, T-1035-1047 (1960)

[4] P.P. Townend, T.W. Chw; Text. Rec., 74 60-63 (1957)

[5] P.P. Townend, E. Spiegel; J. Text.Inst.,37, T58-76 (1946)

[6] W.J. Byatt, J.P. Elting; Text.Res.J.,28,417-421(1958)

[7] W.J. Byatt; Text. Res.J. 31,171-174 (1961)

[8] R. Meyer, K.K. Almin, B. Steenberg; Brit. J. Appl. Phys., 17, 409-416 (1966)

Note [1]: The relation of $n(l, t)$ and a weight-frequency distribution $w(l, t)$ is

$$
n(l, t)=\frac{w(l, t)}{l}
$$

By the use of this, we can easily convert it, the relation obtained in this article, into the expression with the weitgh frequency distribution.

For instance, eq. (2) transforms thus:

$$
\begin{aligned}
w(l, t) & =-\alpha(l) w(l, t) \\
& +2 l \int_{l_{1}}^{L} \alpha\left(l_{1}\right) \beta\left(l_{1} \rightarrow l\right) \frac{1}{l_{1}} w\left(l_{1}, t\right) d l_{1}
\end{aligned}
$$

Note [2]: Eq. (32) is deducible directly from eq. (2). Integrating eq. (2) from $l$ to $L$, since the integration of the 2 nd term becomes zero, the following obtainable:

$$
\frac{\partial N(t)}{\partial t}=\int_{0}^{L} \alpha(l) n(l, t) d l
$$

As $n(l, t) \geq 0$, therefore, we can find such $\xi$ to be $0 \leq$ $\xi \leq L$ as being satisfied as follows:

$$
\frac{\partial N(t)}{\partial t}=\alpha(\xi) N(t)
$$

Solving this, the following upper limit for the total number of fibers is obtainable:

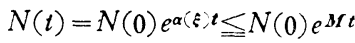

